\title{
Guest editorial to the theme issue on traceability in model-driven engineering
}

\author{
Richard F. Paige - Goran K. Olsen • Jon Oldevik • \\ Tor Neple
}

Published online: 27 February 2010

(C) Springer-Verlag 2010

This issue of Software and Systems Modeling, and part of the issue that follows, are dedicated to the theme of traceability in model-driven engineering (MDE). Traceability is a fundamental concern in MDE processes, where models are related via application of different model management operations, such as model-to-model transformations, modelto-text transformations, model merging, model comparison, and many others. MDE emphasises on the application of automated model management operations, and substantial traceability information can be produced as a side-effect of applying these operations. In addition, in realistic MDE processes, traceability information can be produced by hand, through engineers manually relate MDE artefacts, or relate MDE artefacts with other artefacts (such as requirements documents). Overall, there are many challenges to traceability in MDE, ranging from managing large traceability models, to synchronizing models, and to keep traceability information consistent when models are being modified automatically and manually. This theme issue presents state-ofthe-art research on these and other challenges.

Since 2005, the ECMDA-FA/ECMFA Workshop on Traceability has presented the leading research on traceability in MDE. After the 2008 workshop in Berlin, authors of accepted papers from the previous four workshops were invited to submit their latest results (perhaps derived from their workshop papers) to this theme issue. At the same time, an open call was issued, soliciting submissions from the

\section{R. F. Paige $(\bowtie)$}

University of York, York, UK

e-mail: paige@cs.york.ac.uk

G. K. Olsen · J. Oldevik

SINTEF, Oslo, Norway

T. Neple

Norse Solutions, Lysaker, Norway research community. A total of 13 papers were submitted. All submitted papers underwent a full review process, and were evaluated by at least three reviewers, including some of the original program and organising committee members of the Workshops on Traceability, as well as new expert reviewers. Seven papers were finally selected for inclusion in the journal.

The first five papers are included in this issue, and the remaining two appear in the next issue.

"A Model-Driven Traceability Framework for Software Product Lines" by Nicolas Anquetil, Uira Kulesza, Ralf Mitschke, Ana Moreira, Jean-Claude Royer, Andreas Rummler and André Sousa describes a new approach to managing traceability information for models of software product lines which deals with the challenges associated with configuration and parametrization.

"Synchronization of Abstract and Concrete Syntax in Domain-Specific Modeling Languages by Advanced Mapping Models and Live Transformations" by Istvan Rath, András Ókrós and Dániel Varró presents a novel approach to the challenging problem of synchronizing concrete and abstract syntax, via application of relationships between models, operationalized by live transformations.

"Graph-Based Traceability-A Comprehensive Approach” by Hannes Schwarz, Júrgen Ebert and Andreas Winter presents an approach that aims to support a full range of traceability activities (such as trace establishment) via graph technology.

"Dynamic Hierarchical Mega Models-Comprehensive Traceability and Efficient Maintenance” by Andreas Seibel, Stefan Neumann and Holger Giese describes a way of combining traditional approaches to traceability with global model management technology and shows how to efficiently manage traceability information via the so-called dynamic hierarchical megamodels. 
"A Survey of Traceability in Requirements Engineering and Model Driven Development" by Stefan Winkler and Jens von Pilgrim, as the title suggests, presents a comprehensive and up-to-date survey of traceability research, solutions and tools in the area of MDE and requirements engineering. The authors also present a gap analysis that describes unresolved challenges affecting both domains.

The following two papers appear in the next issue of SoSyM.

"VbTrace: Using View-based and Model-driven Development to Support Traceability in Process-driven SOAs" by Huy Tran, Uwe Zdun and Schahram Dustdar explores traceability in a novel domain: process-driven ServiceOriented Architectures. It describes a novel traceability mechanism based on the notion of views encoded as models.

Finally, "Semantics of Trace Relations in Requirements Models for Consistency Checking and Inferencing” by Arda Goknil, Ivan Kurtev, Klaas van den Berg and Jan-Willem Veldhuis presents mathematical definitions of trace relations to enable analysis. The authors illustrate how the mathematical definitions can be applied to calculate new relations via examples and also present prototypical tool support. 